\title{
Cerebral syphilitic gumma masquerading as cerebral metastatic tumors: case report
}

\author{
${ }^{*}$ Chuanbo Weng, MA, ${ }^{1}$ Kebing Huang, MA, ${ }^{2}$ Tao Jiang, MD, ${ }^{1,2}$ Gege Zhou, MA,, ${ }^{3,4}$ and \\ Tingmiao $\mathrm{Wu}, \mathrm{MA}^{5}$
}

\begin{abstract}
'Department of Neurosurgery, Fourth Affiliated Hospital of Anhui Medical University, Hefei; ${ }^{2}$ School of Neurosurgery, Anhui Medical University, Hefei; ${ }^{3}$ Department of Pathology, Second Affiliated Hospital of Anhui Medical University, Hefei; ${ }^{4}$ Department of Pathology, People's Hospital of Ningguo; and 5Department of Imaging, Fourth Affiliated Hospital of Anhui Medical University, Hefei, Anhui Province, China
\end{abstract}

\begin{abstract}
A 45-year-old man was admitted with severe headache and left-sided weakness, which worsened over 1 week. Brain imaging revealed a small lesion close to the sagittal sinus in the right frontal lobe with severe perilesional edema and showed enhancement on both CT and MRI obtained with contrast. Serological findings were positive for toluidine red unheated serum test (TRUST) positivity and Treponema pallidum particle agglutination assay. The patient was first suspected of having a malignant brain tumor and subsequently received emergency craniotomy. Brain biopsy suggested a diagnosis of syphilitic cerebral gumma; meanwhile the postoperative CSF TRUST titer was positive, and the patient's improvement with high-dose intravenous aqueous crystalline penicillin further supported this etiology. Finally, the lesion on the right frontal lobe had disappeared during the follow-up imaging examination and the myodynamia of the left limbs gradually improved. The authors recommend that diagnostic penicillin treatment should be first implemented. When a patient's history, clinical manifestations, syphilis serology, CSF examination, and other physiological changes indicate a diagnosis of syphilitic cerebral gumma, there is no doubt that surgery should be performed in patients with acute intracranial hypertension, but unnecessary craniotomy should be avoided as far as possible.
\end{abstract}

https://thejns.org/doi/abs/10.3171/2019.5.FOCUS1953

KEYWORDS neurosyphilis; syphilitic cerebral gumma; myodynamia

$\mathrm{T}$ HE WHO has estimated 5.6 million new cases of syphilis each year, with 17.7 million people between the ages of 15 and 49 years worldwide suffering from syphilis in 2012 according to the most recent estimation. The estimated prevalence and incidence of syphilis varies by region and country, with Africa having the highest prevalence, and low- and middle-income countries having more than $60 \%$ of new cases. ${ }^{6}$ The frequency of syphilis was constantly changing during different decades. Due to the widespread use of penicillin, syphilis infection had been declining from the 1950s through the 1990s, but from the 1990s to the present, syphilis has shown a sharp upward trend worldwide. ${ }^{11}$ The United States has mirrored the global trend with a sharp increase in syphilis: in the 1950 s the number of cases in the United States decreased significantly, with only approximately 6500 cases report- ed each year, but in the early 1990s there were between 19,000 and 26,000 cases each year. ${ }^{3}$ Men who have sex with men, coinfection of HIV, and sharing of needles between drug users were probably major reasons behind the resurgence of syphilis.?

The diagnosis of syphilis is usually based on a suggestive clinical history and supportive serological examination. Serological examination for syphilis can be broadly divided into nontreponemal tests (NTTs) and treponemal tests (TTs). The most commonly used NTTs include the rapid plasma regain (RPR) test and the toluidine red unheated serum test (TRUST), which measure immunoglobulins produced in response to lipoidal material released from the bacterium and/or dying host cell. Compared to NTTs, in theory TTs (e.g., Treponema pallidum particle agglutination [TPPA]) have high specificity for detect-

ABBREVIATIONS DWI = diffusion-weighted imaging; NTT = nontreponemal test; TPPA = Treponema pallidum particle agglutination; TRUST = toluidine red unheated serum test; TT = treponemal test.

SUBMITTED January 28, 2019. ACCEPTED May 1, 2019

INCLUDE WHEN CITING DOI: 10.3171/2019.5.FOCUS1953.

${ }^{*}$ C.W. and K.H. have contributed equally to this work and share first authorship. 
ing antibodies against $T$. pallidum proteins. After effective treatment, the levels of nontreponemal antibodies decrease, whereas the levels of treponemal antibodies remain high for many years. So, NTTs are useful in evaluating treatment effectiveness (detecting active syphilis). TTs are usually used for confirmatory analysis after a positive NTT result. Neurosyphilis is an important diagnosis but is often neglected-partially because most patients with early syphilis who have CSF abnormalities have no CNS symptoms. ${ }^{4}$ Because it is a rarity with diverse characteristics on imaging, cerebral syphilitic gumma is often misdiagnosed and easily confused with brain tumor.

Here, we report an interesting and unusual case of an adult male patient with cerebral syphilitic gumma who presented with a large intracranial lesion in the right frontal lobe masquerading as a malignant brain tumor. We also present an extensive review of the literature to better characterize cerebral syphilitic gumma.

\section{Case Report}

History and Examination

The patient was a 45-year-old right-handed man who was admitted to our hospital on October 25, 2018. He was a long-distance truck driver with no significant past medical history. Before presentation, he had a 10-day history of severe headache with a progressive decrease in muscle strength of his left limbs. At admission, the patient had clear consciousness, with small areas of red rash on the skin, normal right-limb muscle strength (Medical Research Council grade 5), but decreased muscle strength in the left limbs (Medical Research Council grade 4).

Examination of the blood at admission showed the following results: TRUST positivity, TPPA assay positivity, and hepatitis B virus surface antigen positivity-other blood testing showed no abnormality (Table 1). A CSF examination was executed after histopathological confirmation. CSF routine examination and biochemical indexes were basically normal, but TPPA was positive. Of specific interest here was the fact that the CSF TRUST titer was the same as blood at admission and before discharge (Table 1).

\section{Neuroimaging}

CT scanning of the brain showed a small low-density lesion close to the sagittal sinus in the right frontal lobe with severe perilesional edema. Slight homogeneous enhancement was seen on the CT scan obtained with contrast (Fig. 1A and B).

Head MRI showed a slight isointensity on T1 and a long T2 nodular signal shadow adjacent to the right cerebral falx, as well as a somewhat high T2-FLAIR and significantly higher diffusion-weighted imaging (DWI) signal, which measured approximately $1.8 \mathrm{~cm} \times 1.7 \mathrm{~cm}$ $\times 2.2 \mathrm{~cm}$, surrounded by severe edema around the lesion. After addition of contrast, the lesion presented as ring enhanced and the adjacent meninges were slightly thickened and enhanced (Fig. 1C-H).

\section{Treatments}

After hospitalization, the patient's myodynamia of the left limbs gradually worsened, which might be asso-
TABLE 1. Laboratory examination parameters in a patient with neurosyphilis

\begin{tabular}{lccc}
\hline \multicolumn{1}{c}{ Parameter } & At Admission & Before Discharge & At Follow-Up \\
\hline White blood cells & $5.32 \times 10^{9} / \mathrm{L}$ & $7.84 \times 10^{9} / \mathrm{L}$ & $3.67 \times 10^{9} / \mathrm{L}$ \\
\hline Neutrophil rate & $73.5 \%$ & $71.0 \%$ & $57.8 \%$ \\
\hline Neutrophil count & $3.91 \times 10^{9} / \mathrm{L}$ & $5.56 \times 10^{9} / \mathrm{L}$ & $2.13 \times 10^{9} / \mathrm{L}$ \\
\hline Red blood cells & $4.77 \times 10^{12} / \mathrm{L}$ & $4.27 \times 10^{12} / \mathrm{L}$ & $4.63 \times 10^{12} / \mathrm{L}$ \\
\hline Platelet count & $241 \times 10^{9} / \mathrm{L}$ & $193 \times 10^{9} / \mathrm{L}$ & $190 \times 10^{9} / \mathrm{L}$ \\
\hline TP-Ab & + & Not tested & Not tested \\
\hline TRUST & $1: 16$ & $1: 8$ & $1: 8$ \\
\hline TPPA & + & Not tested & Not tested \\
\hline HBsAg & + & Not tested & Not tested \\
\hline CSF white blood & $0.003 \times 10^{9} / \mathrm{L}$ & $0.003 \times 10^{9} / \mathrm{L}$ & Not tested \\
cells & & & \\
\hline CSF glucose & $4.63 \mathrm{mmol} / \mathrm{L}$ & $4.21 \mathrm{mmol} / \mathrm{L}$ & Not tested \\
\hline CSF Cl & $118.4 \mathrm{mmol} / \mathrm{L}$ & $130.7 \mathrm{mmol} / \mathrm{L}$ & Not tested \\
\hline CFP & $301 \mathrm{mg} / \mathrm{L}$ & $379 \mathrm{mg} / \mathrm{L}$ & Not tested \\
\hline PTS & - & - & Not tested \\
\hline CSF TRUST & $1: 16$ & $1: 8$ & Not tested \\
\hline
\end{tabular}

$\mathrm{CFP}=\mathrm{CSF}$ protein; $\mathrm{HBsAg}=$ hepatitis B surface antigen; PTS = Pandy test; $\mathrm{TP}-\mathrm{A} \mathrm{b}=\mathrm{T}$. pallidum antibody; + = positive; $-=$ negative .

ciated with severe perilesional edema according to CT scanning. With a provisional diagnosis of glioblastoma or metastatic brain tumor after MR scanning, we performed an emergency craniotomy and the lesion was resected completely (Fig. 2). Consistent with the rapid intraoperative pathological results, histopathological examination showed multiple coagulative necroses; a large quantity of inflammatory cell infiltration (lymphocytes, plasma cells, and neutrophils); angiectasis; proliferation of fibroblasts; and inflammatory changes of the meningeal tissue (Fig. 2). Postoperative examination of CSF indicated that the titer of TRUST was 1:16 and there was TPPA positivity. Finally, the patient was diagnosed with cerebral syphilitic gumma according to CSF findings and pathological examinations. Consequently, he agreed to an antisyphilitic treatment, which included a 14-day course of intravenous penicillin ( 2.5 million $U$ administered every 4 hours) followed by a 3-week course of intramuscular injections of benzathine penicillin ( 2.4 million $\mathrm{U}$ administered once per week). ${ }^{12}$

\section{Postoperative Course}

At the follow-up visit 1 month after surgery, the patient's myodynamia of the left limbs gradually improved and the right frontal lobe presented no evidence of lesion recurrence on the imaging examination (Fig. 3), but the blood TRUST titer was still 1:8 even with more intensive penicillin $\mathrm{G}$ treatment (Table 1).

\section{Discussion}

Neurosyphilis can occur throughout the natural history of syphilis infection and has complicated clinical manifestations-including asymptomatic, syphilitic meningitis; meningovascular syphilis; spinal syphilis; and syphilitic 

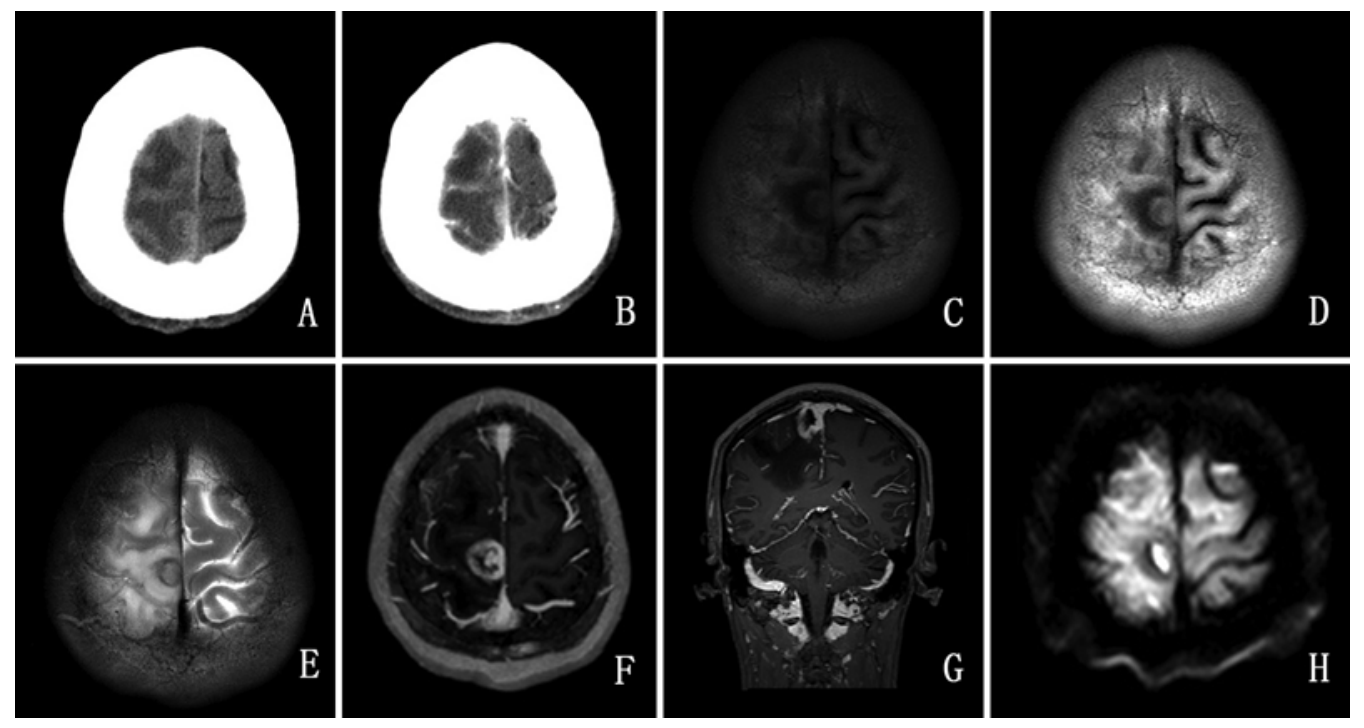

FIG. 1. CT (A), enhanced CT (B), T1-weighted MRI (C), T2-weighted MRI (D), T2-FLAIR MRI (E), enhanced MRI (F and G), and DWI $(\mathbf{H})$. CT showed a low-density lesion adjacent to the cerebral falx with slight enhancement on the CT scan obtained with contrast, and severe perilesional edema. MRI showed the lesion presenting as ring enhanced, and the adjacent meninges were slightly thickened and enhanced.

gumma-but isolated or multiple intracranial gummous lesions are rare, perhaps as a result of the introduction of penicillin therapy for early syphilis. ${ }^{1,4}$ However, surveys indicate that there are still significant numbers of patients with cerebral syphilitic gumma, especially in the 18- to 39 -year age group $(48.6 \%)$, and that these lesions are most common on the convexities $(66 \%){ }^{2}$ Because of the low incidence, misdiagnosis is the trickiest clinical problem for syphilitic gumma, often because of diverse characteristics on imaging. The syphilitic gumma could be easily misdiagnosed as a brain tumor, such as meningioma or malignant lymphoma, and in this patient we confused the lesion with metastatic brain tumor at first. So, most cerebral syphilitic gumma are finally diagnosed by pathological confirmation after the operation.
It is known that the diagnosis of cerebral syphilitic gumma from radiological testing is a challenge because often imaging findings can vary. In most cases the lesions were multiple, with irregular enhancement and mild surrounding edema. ${ }^{2,8}$ In our case there was a large area of edema around the lesion, which caused a space-occupying effect. The lesion showed uncommon ring enhancement, which is rarely reported in the literature. We hypothesize that necrosis at the nodule center of the gumma surrounded by epithelial cells, multinucleated giant cells, lymphocytes, and granulation tissue may be responsible for this ring enhancement. Extensive edema usually surrounded the lesions due to the chronic inflammatory processes of syphilis. It should also be noted that cerebral syphilitic gumma could encroach on and is closely related to the
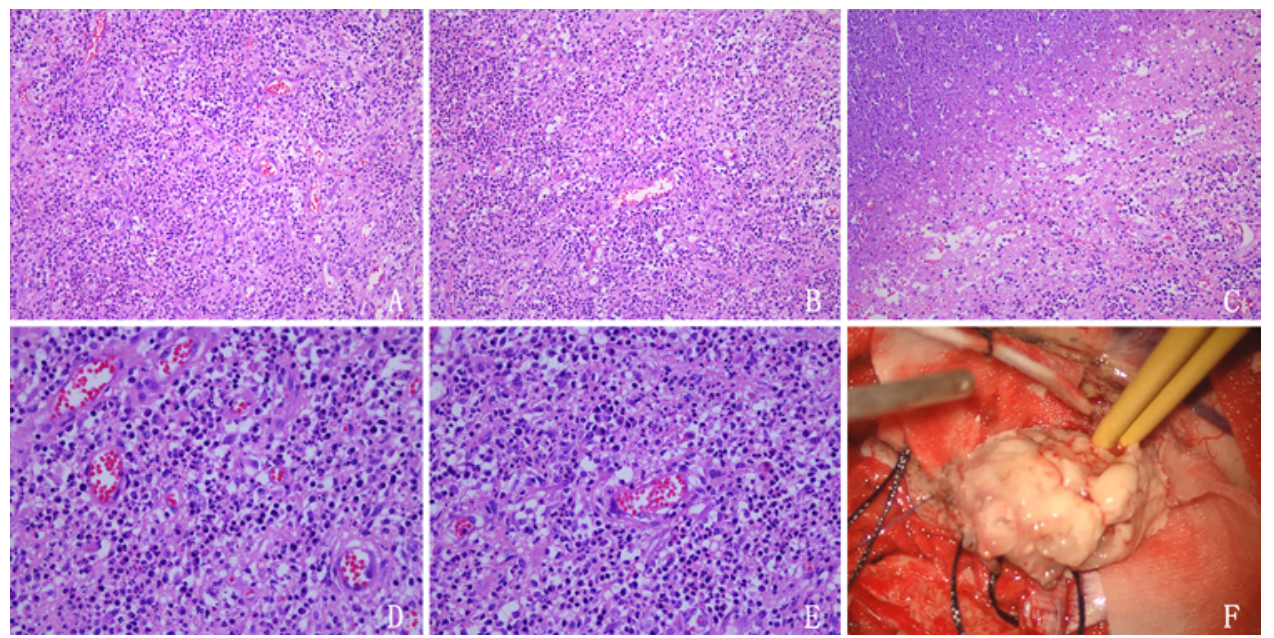

FIG. 2. Histopathological examination showed a large quantity of inflammatory cell infiltration containing lymphocytes, neutrophils, and necrosis $(A-E)$. H \& E, original magnification $\times 100(A-C)$ and $\times 200(D$ and $E)$. The lesion was gray-white, irregular in shape, and had a slightly rubbery texture $(\mathbf{F})$. 

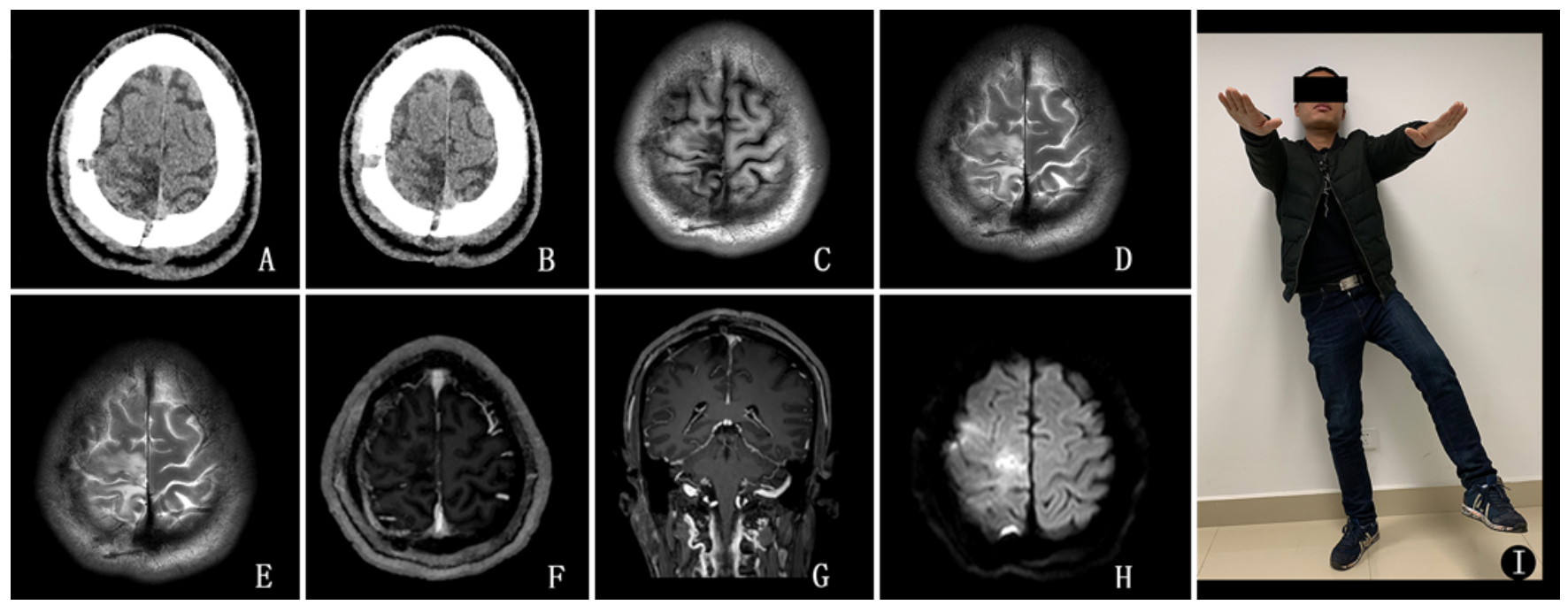

FIG. 3. CT (A and B), T1-weighted MRI (C), T2-weighted MRI (D), T2-FLAIR MRI (E), enhanced MRI (F and G), and DWI (H). At the 1-month follow-up, the imaging examination indicated that there was no lesion recurrence on the right frontal lobe and the patient's myodynamia of the left limbs gradually improved (I).

meninges, causing significant meningeal enhancement similar to those for meningioma. Auxiliary examinations that are helpful in the diagnosis of neurosyphilis included syphilis serological tests of the CSF. Analysis of CSF in patients with active neurosyphilis usually produces a positive Venereal Disease Research Laboratory (VDRL) test, elevated protein, and elevated white blood cell count. ${ }^{10}$ In our patient, CSF routine examination and biochemical indexes were basically normal, elevated protein and elevated white blood cell count were not noted, but TPPA was positive. Syphilis serological tests of the CSF could confirm a diagnosis of active neurosyphilis by combined analysis of a patient's history. Considering the diagnosis of neurosyphilis gumma, patients can attain verification of diagnostic accuracy by antisyphilis treatment, which will help to avoid unnecessary craniotomy.

As the mainstay of treatment for syphilis, intramuscular injection of long-acting benzathine penicillin $\mathrm{G}$ can effectively maintain penicillin levels in the blood above the minimum inhibitory concentration for $\geq 10$ days. A lumbar puncture and CSF examination should be executed in patients with symptoms indicating neurosyphilis to confirm or exclude this diagnosis. Once diagnosed, large doses of intravenous injection of crystalline penicillin or intramuscular injection of procaine penicillin plus probenecid are required for 10-14 days. ${ }^{5}$ It has been established in the literature that syphilitic gumma could apparently be absorbed or could disappear after penicillin treatment., Therefore, patients with cerebral syphilitic gumma can receive this diagnostic treatment to avoid unnecessary craniotomy. Penicillin treatment efficacy is measured indirectly by using serology, and a cure is usually defined as reversion to a negative serum status or a 4-fold reduction in the titer from an NTT. However, a minority of patients remain seropositive and their NTT titers show a less than 4-fold reduction, although they have almost certainly been cured, and there is no evidence of progressive disease-the socalled syphilitic sero-fixation. In our case, after penicillin treatment, no recurrence of syphilitic gumma was found by imaging examination, but the TRUST titer remained positive at 1:8 and no further decrease was observed.

\section{Conclusions}

We present a patient with hemiplegia who was subsequently diagnosed with syphilitic cerebral gumma after postoperative pathological examination. It has been reported that cerebral syphilitic gumma could be cured by high-dose intravenous aqueous crystalline penicillin, but the real question was whether it could be correctly diagnosed. It was difficult to diagnose cerebral syphilis gumma by imaging examination, because it had a similar imaging appearance to many other intracranial lesions. Therefore, the diagnosis of cerebral syphilitic gumma required a consideration of the patient history, clinical manifestations, syphilis serology, CSF examination, and other physiological changes-i.e., not just imaging. Our recommendations were that cerebral syphilitic gumma should be treated with intravenous penicillin $\mathrm{G}$ as the preferred therapy and that the physician try to avoid craniotomy unless the patient has symptoms of raised intracranial pressure.

\section{Acknowledgments}

This manuscript was written with the help of many other colleagues: Xukou Wang, Ye Zhou, Yang Wan, Lulu Yang, Hao Dong, and Tingsheng Zhao. Among them, special thanks should be given to Yang Wan for his help in the collection of pathological data. We also received the help of Dr. Ye Zhou for the treatment of neurosyphilis with penicillin.

\section{References}

1. Drago F, Ciccarese G, Merlo G, Sartoris G, Parodi A: Is the standard treatment for early syphilis sufficient to prevent cardiovascular and neurologic syphilis? Am J Cardiol 117:310-311, 2016

2. Fargen KM, Alvernia JE, Lin CS, Melgar M: Cerebral 
syphilitic gummata: a case presentation and analysis of 156 reported cases. Neurosurgery 64:568-576, 2009

3. Hook EW III, Marra CM: Acquired syphilis in adults. $\mathbf{N}$ Engl J Med 326:1060-1069, 1992

4. Jiang Y, Zhang YF, Liu M, Ma LL, Peng FH, Huang QL, et al: Syphilitic dementia and lipid metabolism. Eur J Neurol 23:1541-1547, 2016

5. Kingston M, French $P$, Higgins $S$, McQuillan O, Sukthankar A, Stott C, et al: UK national guidelines on the management of syphilis 2015. Int J STD AIDS 27:421-446, 2016

6. Newman L, Rowley J, Vander Hoorn S, Wijesooriya NS, Unemo M, Low N, et al: Global estimates of the prevalence and incidence of four curable sexually transmitted infections in 2012 based on systematic review and global reporting. PLoS One 10:e0143304, 2015

7. Seña AC, Zhang XH, Li T, Zheng HP, Yang B, Yang LG, et al: A systematic review of syphilis serological treatment outcomes in HIV-infected and HIV-uninfected persons: rethinking the significance of serological non-responsiveness and the serofast state after therapy. BMC Infect Dis 15:479, 2015

8. Shao X, Qiang D, Liu Y, Yuan Q, Tao J, Ji B: Diagnosis and treatment of cerebral syphilitic gumma: a report of three cases. Front Neurosci 12:100, 2018

9. Stamm LV: Global challenge of antibiotic-resistant Treponema pallidum. Antimicrob Agents Chemother 54:583-589, 2010

10. Timmermans M, Carr J: Neurosyphilis in the modern era. J Neurol Neurosurg Psychiatry 75:1727-1730, 2004
11. Tucker JD, Cohen MS: China's syphilis epidemic: epidemiology, proximate determinants of spread, and control responses. Curr Opin Infect Dis 24:50-55, 2011

12. Workowski KA: Centers for Disease Control and Prevention Sexually Transmitted Diseases Treatment Guidelines. Clin Infect Dis 61 (Suppl 8):S759-S762, 2015

\section{Disclosures}

The authors report no conflict of interest concerning the materials or methods used in this study or the findings specified in this paper.

\section{Author Contributions}

Conception and design: Jiang, Weng. Acquisition of data: all authors. Drafting the article: Weng, Huang. Critically revising the article: Jiang, Weng. Reviewed submitted version of manuscript: Jiang, Weng, Huang. Approved the final version of the manuscript on behalf of all authors: Jiang. Administrative/technical/material support: Jiang, Zhou, Wu.

\section{Correspondence}

Tao Jiang: Department of Neurosurgery, Fourth Affiliated Hospital of Anhui Medical University, Hefei, Anhui Province, China. jiangtao_aydsfy@163.com. 